\title{
Associations between Demographics and Quality of Life in Children in the First Year of Cancer Treatment
}

\author{
Jennifer Raybin ${ }^{1}$, Verna Hendricks-Ferguson ${ }^{2}$, Paul Cook ${ }^{3}$, and Catherine Jankowski ${ }^{3}$ \\ ${ }^{1}$ Children's Hospital Colorado \\ ${ }^{2}$ Saint Louis University School of Nursing \\ ${ }^{3}$ University of Colorado - Anschutz Medical Campus
}

July 27, 2021

\begin{abstract}
Symptom distress and decreased quality of life (QOL) among children with cancer are well documented. Research is emerging on the child's voice in QOL-symptom reports, but existing QOL questionnaires are burdensome and objective biologic markers are lacking. We examined children's symptoms and QOL from parent and child perspectives and compared the results to one biologic marker (body posture). A cross-sectional secondary analysis of prospective data from children receiving creative arts therapy explored potential associations among demographics with and between QOL measures (PedsQL, Faces Scale, posture). Children $(\mathrm{n}=98)$ ranged in age from 3-17 years $(\mathrm{M}=7.8)$ and were in the first year of cancer treatment. No significant associations were found among the child's sex, race/ethnicity, socioeconomic status (SES), or distance from hospital and total PedsQL. Older age was associated with worse total PedsQL, pain, nausea, worry, and posture (all ps < .05). Greater worry $(\beta=0.51)$ and worse posture $(\beta=0.41)$ were the QOL variables most strongly correlated with older age. Poorer posture was associated with worse child PedsQL (total score, nausea, treatment anxiety, cognitive) and parent PedsQL (pain, nausea). Worse scores on the Faces Scale, PedsQL, and posture were all correlated ( $\mathrm{rs}=.21-.39$, all ps $<.05$ ). Interventions to improve QOL could target nausea, worry, and older patients. Accuracy and interpretation of symptom distress in children is problematic. The Faces Scale and posture may be suitable, readily obtained measures of QOL in pediatric oncology that hold promise.
\end{abstract}

\section{Associations between Demographics and Quality of Life in Children in the First Year of Cancer Treatment}

Jennifer L. Raybin, PhD, RN, CPNP ${ }^{1,2}$; Verna Hendricks-Ferguson, PhD, RN, FPCN, FAAN ${ }^{3}$; Paul Cook, $\mathrm{PhD}^{2}$; Catherine Jankowski, PhD, FACSM ${ }^{2}$

Affiliations: 1. Center for Cancer and Blood Disorders, Children's Hospital Colorado; 2. University of Colorado Anschutz Medical Campus College of Nursing and School of Medicine; 3. Saint Louis University School of Nursing

Word count: Abstract 245, Text 3086

Number of Tables: 3

Running title: Demographics and QOL in pediatric cancer

Key Words: Pediatric, cancer, quality of life, demographics, posture

Abbreviations: 


\begin{tabular}{ll}
\hline QOL & Quality of life \\
\hline SES & Socioeconomic status \\
CAT & Creative arts therapy \\
PI & Principal investigator \\
ICC & Intraclass correlation coefficient \\
CI & Confidence interval \\
ANOVA & Analysis of variance \\
SE & Standard error \\
CNS & Central nervous system \\
\hline
\end{tabular}

Previously published as a meeting abstract:

Raybin, JL, Hendricks-Ferguson, V, Krajicek, M, Cook, P, Jankowski, C. (2021).

Associations between demographic and quality of life in children in the first year of

cancer treatment. [Abstract] NINR ONS NCI Symptom Science Symposium. Oncology Nursing Forum, $48(2): 36$.

\begin{abstract}
Symptom distress and decreased quality of life (QOL) among children with cancer are well documented. Research is emerging on the child's voice in QOL-symptom reports, but existing QOL questionnaires are burdensome and objective biologic markers are lacking. We examined children's symptoms and QOL from parent and child perspectives and compared the results to one biologic marker (body posture). A crosssectional secondary analysis of prospective data from children receiving creative arts therapy explored potential associations among demographics with and between QOL measures (PedsQL, Faces Scale, posture). Children $(n=98)$ ranged in age from 3-17 years $(M=7.8)$ and were in the first year of cancer treatment. No significant associations were found among the child's sex, race/ethnicity, socioeconomic status (SES), or distance from hospital and total PedsQL. Older age was associated with worse total PedsQL, pain, nausea, worry, and posture (all ps $<.05)$. Greater worry $(\beta=0.51)$ and worse posture $(\beta=0.41)$ were the QOL variables most strongly correlated with older age. Poorer posture was associated with worse child PedsQL (total score, nausea, treatment anxiety, cognitive) and parent PedsQL (pain, nausea). Worse scores on the Faces Scale, PedsQL, and posture were all correlated ( $\mathrm{rs}=.21-.39$, all $\mathrm{ps}<.05)$. Interventions to improve QOL could target nausea, worry, and older patients. Accuracy and interpretation of symptom distress in children is problematic. The Faces Scale and posture may be suitable, readily obtained measures of QOL in pediatric oncology that hold promise.
\end{abstract}

\title{
Introduction
}

Symptom distress and decreased quality of life (QOL) has been well documented among children with cancer, ${ }^{1}$ but little is known regarding the demographic characteristics that could be targeted for interventions. The few studies in the literature examining demographic differences in the QOL of children with cancer are limited to children with leukemia, ${ }^{2-4}$ children with advanced cancer, ${ }^{5,6}$ and survivors of childhood cancer. ${ }^{7}$ Although the results are mixed, it appears that female gender and younger age are related to worse QOL, and older age is associated with more nausea..$^{2,5-7}$ Thus, we sought to examine sex, race/ethnicity, age, and socio-economic (SES) differences in QOL in children in the first year of treatment for any type of cancer. We used questionnaires and a novel objective biologic measure to examine QOL.

QOL is usually measured with questionnaires in children with cancer. This practice is inherently limited by subjectivity, age, and developmental stage. An objective measure could reduce the time and possible distress associated with questionnaires and more efficiently and accurately determine the outcomes of interventions to improve QOL. ${ }^{8,9}$ Systematic review of standing posture measured as thoracic kyphosis shows promise as a marker of QOL (manuscript under review). Indeed, posture has been studied in survivors of pediatric cancer 
as an outcome variable of neurologic injury, but not for overall QOL. ${ }^{10,11}$ In terms of the variables that may affect posture, studies have examined adults and healthy children. Posture did not differ by sex in older adults with kyphosis ${ }^{12}$ but did differ by age ${ }^{13}$ and sex in healthy children ${ }^{14,15}$ however this relationship has not been examined in children with cancer.

Disparities in SES, race, and ethnicity can no longer be ignored in health care research. New work is emerging on the association of financial burden with worse QOL in children newly diagnosed with cancer ${ }^{16}$ children undergoing stem cell transplant,${ }^{17}$ children with advanced cancer,,${ }^{6,18}$ and children who have completed cancer therapy. ${ }^{19}$ In our data set, education level of parent and zip code were included as surrogate variables for SES. ${ }^{20}$ Race and ethnicity have been studied as predictors of overall survival in various pediatric cancers, ${ }^{21,22}$ QOL of children with leukemia, ${ }^{23}$ experiences in end-of-life care, ${ }^{24}$ and QOL of childhood cancer survivors. ${ }^{25}$ Less is known of SES as a predictor of QOL in children in early treatment for any type of cancer.

Finally, although the Faces Scale for pain ${ }^{26}$ has been validated to measure nausea, ${ }^{27}$ fatigue, ${ }^{28}$ and anxiety, ${ }^{29}$ it is not known if it can be used as a surrogate for global QOL.

To evaluate the inter-relationships of demographic variables, measures of QOL, and posture, a cross-sectional secondary analysis of a study including 100 children with cancer was completed to inform potential targets for future intervention studies. An exploratory analysis was also used to query the data for potential relationships between posture, the Faces Scale, and the PedsQL Cancer scales.

\section{Methods}

A secondary analysis was conducted to examine the associations of a) baseline QOL, Faces, and posture with age, sex, race, SES, geographic location; and b) baseline posture, Faces, and PedsQL Cancer scales with each other.

\section{Research Aims and Hypotheses}

We aimed to examine associations of demographic variables with QOL in children with cancer. We predicted that sex and age differences existed in the QOL (PedsQL, Faces Scale, posture) of these children during cancer treatment. Race, ethnicity, education level, and zip code (urban/rural) "distance" differences also were predicted to be present in this population. We hypothesized that younger age would be associated with more erect posture and that sex differences existed in posture in children receiving cancer treatment. Additionally, we aimed to explore the correlations of the Faces Scale, Posture, and the PedsQL with each other and predicted that happier faces, higher QOL and more erect posture would all be correlated.

\section{Population and Sample}

The original longitudinal prospective study examined the QOL of children with cancer receiving Creative Arts Therapy (CAT) and was conducted at a large pediatric cancer center in the western United States treating approximately 250 new oncology patients annually. The study was approved by the university's Institutional Review Board and the cancer center's Protocol Review and Monitoring System. About half of the new patients each year were anticipated to fit the inclusion criteria. Eligibility criteria for inclusion in the original study were a) English-speaking oncology patients (due to the use of published study tools), b) ages 3 to 18 years, and c) currently receiving chemotherapy. Patients who had previously attended two or more CAT sessions were excluded.

Eligible patients were identified by the study team. The study was explained to the patient/family, and the principal investigator (PI) or the research assistant obtained informed consent from the caregiver and informed assent from children over 7 years of age. Further details of the original study are reported elsewhere (manuscript under review).

\section{Instrumentation}

The data set previously entered in Research Electronic Data Capture system (REDCap) ${ }^{30,31}$ was used to examine cross-sectional relationships between demographic variables and study outcomes at baseline. 
Variables included QOL questionnaires, the Faces Scale, as well as physical posture measurements with an inclinometer. Demographic data included in the data base were sex, age, race/ethnicity, type of cancer, type of cancer treatment, educational level of parents, and zip code.

\section{PedsQL 3.0 Cancer Module}

The PedsQL 3.0 Cancer Module evolved from the PedsQL 4.0 Generic Core Scale. ${ }^{32}$ The instrument employs a Parent-Proxy Report for ages 2-18 years and a companion Child Self-Report for children aged 5 years and older. The scores range from 0 to 100 with a larger score indicating better QOL. Validity and reliability testing for both the Core Scale and Cancer Module have been extensive. The Cancer Module is a 27-item instrument that includes 8 subscales (pain and hurt, nausea, procedural anxiety, treatment anxiety, worry, cognitive problems, perceived physical appearance, and communication). The instruments have been successfully employed in several studies of children with cancer. ${ }^{33-35}$ The PedsQL has been shown to be the most responsive to change when compared with other measures of QOL for children undergoing chemotherapy. ${ }^{36,37}$

\section{Faces Scale}

The Faces Scale has been used for evaluating emotional responses to pain in children 3-17 years old. It is a 1-page form with 9 faces in order from "happy" to "upset" with numerical values given to each face. The order of the faces was consistently affirmed by 200 children over 5 years regardless of age, gender, or health status, and the tool has been integrated as a routine measure for management of acute, recurrent, and chronic pain. ${ }^{26,38}$ Although this scale has been used for emotional response to pain, in this study it was also considered an appropriate measure for children's emotional response to discomfort and anxiety in the cancer center.

\section{Posture Measure}

A manual inclinometer was used to measure thoracic kyphosis or standing posture. Reliability and validity of inclinometers have been well documented. Intra- and inter-rater reliability (ICC 0.93-0.97 with 95\% CI 0.88-0.99) was confirmed in subjects with and without symptoms and found measurements of less than 1.7 degrees should be considered measurement error. ${ }^{39-41}$ Validity was supported by change with intervention, and the inclinometer showed good agreement with the gold standard of Cobb angle $(\mathrm{r}=0.86)$, $\mathrm{x}$-rays (ICC $0.89, \mathrm{p}<0.001)$, as well as the photogrammetric technique. ${ }^{42}$ A larger posture angle or score indicates worse posture, and is also hypothesized to indicate greater emotional distress.

\section{Data Analysis}

SPSS version 27 (Armonk NY: IBM Corporation) was used for all analyses. ${ }^{43}$ The data were examined for missingness, normal distribution, and sufficient variance. Pearson correlations were used to assess relationships between normally distributed variables, and Spearman correlations were used as confirmation when one or more of the variables had a distribution that approached non-normality. Linear regressions with ANOVA were used to assess the strength of relationships when initial bivariate screening showed a significant correlation. $T$-tests were used to examine differences between groups based on dichotomous demographic variables. An alpha level of .05 was used as the criterion for statistical significance in all tests. Due to the exploratory and cross-sectional nature of this study, we did not correct for inflated Type I error when performing multiple tests. Results therefore should be regarded as preliminary and as an indicator that a particular relationship merits further study.

\section{Results}

At study entry, 98 records were usable out of the 100 participants who consented for the study. The Faces Scale was completed by all participants (100\%) and the posture measure had a completion rate of $68 \%$. The child report PedsQL had more missing data (59 complete out of 98 participants $=60 \%)$ and was slightly more skewed than the parent proxy PedsQL $(95$ participants completed $=97 \%$ ). The data set was first examined with descriptive analysis to confirm normality, which revealed approximately normal distributions for most 
variables. Only the child report PedsQL subscales of pain, appearance, worry, and treatment anxiety had a skew / $S E$ skew $>2.0$, but these results were still in the 2-3 range, and therefore approximately normally distributed. The child report had a floor effect but no outliers on the high end; therefore, we used parametric tests for the primary analysis, with follow-up nonparametric tests as a sensitivity analysis whenever slight levels of non-normality might have affected the results. Missing data were $<10 \%$ of the total and were handled by case-wise deletion. Although the child report PedsQL had more missing data, only one item was not missing at random (communication) and because of the low rate of missingness this likely did not bias our results. Reliability in this study was acceptable with Cronbach's $\alpha>.90$ on all subscales. Descriptive summary data can be viewed in Table 1.

\section{Demographic Variables Associated with QOL}

There were no differences between males and females on any of the measures or subscales except for the child PedsQL cognitive changes scale, $p=.03$. Older age was significantly correlated with worse scores on the total parent PedsQL and the subscales of nausea, worry, appearance, and communication (Table 2). For the child PedsQL, older age was not correlated with the total score, but was correlated with worse scores on the subscales of pain, nausea, and worry $(p \mathrm{~s}<.05)$. Age was not correlated with the Faces Scale. Race and ethnicity were only correlated with the parent PedsQL cognitive scale $(p=.007)$. SES (parent's highest level of education) was not correlated with either the parent or child PedsQL total or subscale scores. Neither race/ethnicity nor SES were correlated with the Faces Scale or posture. Distance from the hospital in miles was not correlated with any of the measures.

The significant associations found between age and QOL measures were then further examined using linear regression. On the parent report PedsQL, there were small to medium effect sizes for the relationships between age and the total score $\left(F_{1,93}=7.73, p=.007\right.$, standardized $\left.\beta=0.28\right)$, nausea $\left(F_{1,93}=15.71\right.$, $p<.001$, standardized $\beta=0.38)$, appearance $\left(F_{1,93}=15.91, p<.001\right.$, standardized $\left.\beta=0.38\right)$, and communication $\left(F_{1,93}=4.72, p=.03\right.$, standardized $\left.\beta=0.22\right)$. There was also a medium effect size for the relationship between age and worry $\left(F_{1,93}=32.17, p<.001\right.$, standardized $\left.\beta=0.51\right)$. The child report PedsQL also showed small effect sizes for the relationships between age and pain $\left(F_{1,57}=6.05, p=.02\right.$, standardized $\beta=0.31)$, nausea $\left(F_{1,57}=6.78, p=.01\right.$, standardized $\left.\beta=0.33\right)$, and a medium effect size for the relationship between age and worry $\left(F_{1,57}=19.32, p<.001\right.$, standardized $\left.\beta=0.50\right)$. Age also had a moderate effect on posture $\left(F_{1,65}=13.41, p=.001\right.$, standardized $\left.\beta=0.41\right)$.

\section{Comparisons of QOL Measures}

Table 3 shows small to medium effect sizes for the relationships between the three QOL measures. For the parent PedsQL, posture was only correlated with the subscales of pain and nausea. In contrast, the child PedsQL was correlated with the total score, nausea, treatment anxiety, and cognitive scales. On the parent PedsQL, the Faces Scale was correlated with the total score, pain, worry, and appearance. For the child PedsQL, the Faces Scale was also correlated with the total score, pain, and cognitive scales. Posture and the Faces Scale were positively correlated.

\section{Discussion}

These findings confirm our hypothesis that age differences exist in QOL. Older children reported worse quality of life, which is clinically seen with adolescents tolerating cancer treatment worse than toddlers. Our findings contrast with two studies, one in children with leukemia, ${ }^{2}$ and in children with advanced cancer, ${ }^{6}$ in which younger children had worse reported QOL. In the study of children with leukemia, perhaps this difference was related to a younger age range in the study, or because leukemia is more common in young children, whereas our study examined children with all cancer diagnoses. Compared to the findings in the study of children with advanced cancer, our study's findings may reflect higher symptom burden in adolescents in the first year of chemotherapy due to treatment toxicity.

Older children also had less erect posture, which is confirmed by the literature and fits with clinical observations that young children sit and stand straighter than adolescents. However, our hypothesis that 
sex differences exist in posture was not confirmed and differs from published literature. Further study is warranted to explore whether sex differences truly exist in posture.

Pain and nausea are the most prevalent distressing symptoms reported by children with cancer. ${ }^{5,44}$ We also found that age had a moderate relationship with pain and nausea and therefore both should be targeted for symptom management especially in older children. Potential strategies that may appeal to adolescents include integrative modalities that have been shown to improve both pain and nausea in children with cancer. ${ }^{45,46}$

In our study, worry had the strongest relationship with age. This suggests the need for improved treatment to address anxiety through medication management and cognitive behavioral interventions, ${ }^{47}$ particularly for older children. Integrative modalities such as aromatherapy or acupuncture also are known to have usefulness in children with cancer and may be better tolerated in adolescents. ${ }^{48}$

We did not find any other demographic associations with QOL, posture, or Faces Scale scores, which diverged from our hypotheses and from the literature. Other studies have found that girls report worse QOL, but our results showed no differences between girls and boys on either the subjective or the objective QOL measures. Although disparities for minorities have also been reported in QOL for children with cancer, we did not find any differences based on race or ethnicity. However, we collapsed the racial and ethnic subsets into a single dichotomous "minority status" variable due to sample size limitations, a data analysis strategy that could mask results for specific races if these exist.

We found moderately strong relationships between posture, PedsQL, and the Faces Scale, which confirm the idea that all three of these metrics are related to a single underlying construct, QOL. These findings suggest that children who report that they feel better, also stand up straighter, and pick a happier face on the scale. Posture was particularly related to the more physical aspects of the PedsQL such as pain and nausea, which is consistent with the theory of embodiment in which the body stature reflects symptoms and emotions. ${ }^{49}$ Children's report of pain had the strongest relationship with the Faces Scale, which aligns with the original intent of the Faces Scale for pain assessment. ${ }^{26}$ However, the total PedsQL scores for both child and parent report also had moderate relationships with the Faces Scale, a finding that supports further study of this simple tool as a way to measure global QOL in children with cancer.

Likewise, posture and the Faces Scale were moderately related and thus both measures show promise as simple methods to reflect the overall sense of how a child with cancer is feeling. If these relationships can be confirmed and shown to be responsive to change over time, posture and the simplified Faces Scale could potentially be used as surrogates for the more time-consuming QOL questionnaires. Furthermore, the Faces Scale was the only measure that had 100 percent completion which exemplifies the feasibility of this simplified measure in children under stress during cancer treatment.

Additionally, the posture measure could represent a biologic and objective technique that would obviate the subjective limitations of QOL questionnaires. ${ }^{50,51}$ Posture measures have the added benefit of not causing increased pain or distress as seen with blood draws or obtaining cerebrospinal fluid. A posture assessment is even less invasive than obtaining a saliva sample, except the child must feel well enough to stand, which may have been the reason that this measure had the lowest completion rate in our study (68\%). A sitting posture could also be explored for very sick patients. The posture measure could also obviate the limitations of other objective systemic biomarkers such as cortisol that are influenced by the cancer or its treatment.

As a retrospective secondary analysis, this study was powered for the original aims and no correction was used for inflated alpha. Therefore, the current results must be viewed with caution. Although the measures were previously validated and their psychometric characteristics are generally strong, the subscale for cognition on the PedsQL may warrant further examination because it was the only subscale that reflected a significant difference based on sex (parent report) and SES (child report). Selection bias may have existed because children and their parents who chose to participate in the study may have been more likely to do art or were perhaps more interested in QOL. Additionally, the ages of children in this study were skewed toward younger children. Replication of the current results with a larger and more diverse sample of children with 
cancer is therefore needed.

This study advances the field of symptom science in children with cancer. By adding to the literature on demographic associations with QOL, future intervention studies can now target the specific high-risk group of older children. More in-depth study of the relationship of sex and age with posture in children with cancer will also further the investigation of posture as a potential biomarker of QOL. The correlation of the Faces Scale to the PedsQL also supports further study of simplified measures of overall QOL in children with cancer. A prospective multi-site longitudinal study is warranted to further examine posture as an objective measure of QOL in children with cancer.

\section{Conflict of Interest}

None of the authors has financial interests in the subject matter contained in this manuscript. This work was funded in party by American Cancer Society 133523-DSCN-19-050-01-SCN.

\section{Acknowledgements}

We gratefully acknowledge Dr. Marilyn Krajicek for her advisement and careful consideration of this body of work.

\section{References}

1. Rosenberg AR, Wolfe J. Approaching the third decade of paediatric palliative oncology investigation: historical progress and future directions. Lancet Child Adolesc Health. 2017;1(1):56-67.

2. Dupuis LL, Lu X, Mitchell HR, et al. Anxiety, pain, and nausea during the treatment of standard-risk childhood acute lymphoblastic leukemia: A prospective, longitudinal study from the C hildren's O ncology G roup.Cancer. 2016;122(7):1116-1125.

3. Eiser C, Stride C, Vora A, et al. Prospective evaluation of quality of life in children treated in UKALL 2003 for acute lymphoblastic leukaemia: A cohort study. Pediatr Blood Cancer.2017;64(11):e26615.

4. Rae C, Furlong W, Feeny D, et al. Health Status and Health-related Quality of Life Measurement in Pediatric Cancer Clinical Trials: An Examination of the DFCI 00-01 Acute Lymphoblastic Leukemia Protocol.J Pediatr Hematol Oncol. 2018;40(8):580-587.

5. Wolfe J, Orellana L, Ullrich C, et al. Symptoms and Distress in Children With Advanced Cancer: Prospective Patient-Reported Outcomes From the PediQUEST Study. J Clin Oncol. 2015;33(17):19281935.

6. Robson PC, Dietrich MS, Akard TF. Associations of age, gender, and family income with quality of life in children with advanced cancer.J Pediatr Oncol Nurs. 2021:1043454221992321.

7. Halvorsen JF, Sund AM, Zeltzer L, et al. Health-related quality of life and psychological distress in young adult survivors of childhood cancer and their association with treatment, education, and demographic factors. Qual Life Res. 2018;27(2):529-537.

8. Hockenberry MJ, Krull KR, Insel KC, et al. Oxidative Stress, Motor Abilities, and Behavioral Adjustment in Children Treated for Acute Lymphoblastic Leukemia. Oncol Nurs Forum. 2015;42(5):542-549.

9. Moore IM. Advancing biobehavioral research in childhood cancer.J Pediatr Oncol Nurs. 2004;21(3):128131.

10. Einarsson E-J, Patel M, Petersen H, et al. Decreased postural control in adult survivors of childhood cancer treated with chemotherapy. Sci Rep. 2016;6(1):1-10.

11. Varedi M, Lu L, Howell CR, et al. Peripheral neuropathy, sensory processing, and balance in survivors of acute lymphoblastic leukemia.J Clin Oncol. 2018;36(22):2315. 
12. Katzman WB, Parimi N, Gladin A, et al. Sex differences in response to targeted kyphosis specific exercise and posture training in community-dwelling older adults: a randomized controlled trial.BMC Musculoskelet Disord. 2017;18(1):509.

13. Bourelle S, Dey N, Sifaki-Pistolla D, et al. Computerized static posturography and laterality in children. Influence of age. Acta Bioeng Biomech. 2017;19(2):129-139.

14. Araújo FA, Simões D, Silva P, Alegrete N, Lucas R. Sagittal standing posture and relationships with anthropometrics and body composition during childhood. Gait Posture. 2019;73:45-51.

15. Rusek W, Baran J, Leszczak J, et al. The Influence of Body Mass Composition on the Postural Characterization of School-Age Children and Adolescents. Biomed Res Int. 2018;2018:9459014.

16. Bona K, London WB, Guo D, Frank DA, Wolfe J. Trajectory of material hardship and income poverty in families of children undergoing chemotherapy: a prospective cohort study. Pediatr Blood Cancer.2016;63(1):105-111.

17. Bona K, London WB, Guo D, Abel G, Lehmann L, Wolfe J. Prevalence and impact of financial hardship among New England pediatric stem cell transplantation families. Biol Blood Marrow Transplant.2015;21(2):312-318.

18. Ilowite MF, Al-Sayegh H, Ma C, et al. The relationship between household income and patient-reported symptom distress and quality of life in children with advanced cancer: A report from the PediQUEST study. Cancer. 2018;124(19):3934-3941.

19. Bilodeau M, Ma C, Al-Sayegh H, Wolfe J, Bona K. Household material hardship in families of children post-chemotherapy. Pediatr Blood Cancer. 2018;65(1):e26743.

20. Marmot M. The influence of income on health: views of an epidemiologist. Health Aff (Millwood). 2002;21(2):31-46.

21. Kahn JM, Kelly KM, Pei Q, et al. Survival by Race and Ethnicity in Pediatric and Adolescent Patients With Hodgkin Lymphoma: A Children's Oncology Group Study. J Clin Oncol. 2019;37(32):3009-3017.

22. Williams LA, Frazier AL, Poynter JN. Survival differences by race/ethnicity among children and adolescents diagnosed with germ cell tumors. Int J Cancer. 2020;146(9):2433-2441.

23. Nagarajan R, Gerbing R, Alonzo T, et al. Quality of life in pediatric acute myeloid leukemia: Report from the Children's Oncology Group. Cancer Med. 2019;8(9):4454-4464.

24. Kaye EC, Gushue CA, DeMarsh S, et al. Impact of Race and Ethnicity on End-of-Life Experiences for Children With Cancer. Am J Hosp Palliat Care. 2019;36(9):767-774.

25. Dixon SB, Li N, Yasui Y, et al. Racial and ethnic disparities in neurocognitive, emotional, and quality-oflife outcomes in survivors of childhood cancer: A report from the Childhood Cancer Survivor Study.Cancer. $2019 ; 125(20): 3666-3677$.

26. McGrath PA, Seifert CE, Speechley KN, Booth JC, Stitt L, Gibson MC. A new analogue scale for assessing children's pain: an initial validation study. Pain. 1996;64(3):435-443.

27. Baxter AL, Watcha MF, Baxter WV, Leong T, Wyatt MM. Development and validation of a pictorial nausea rating scale for children.Pediatrics. 2011;127(6):e1542-1549.

28. Chuang LL, Lin KC, Hsu AL, et al. Reliability and validity of a vertical numerical rating scale supplemented with a faces rating scale in measuring fatigue after stroke. Health Qual Life Outcomes.2015;13:91.

29. Bellon M, Taillardat E, Horlin AL, et al. Validation of a simple tool for anxiety trait screening in children presenting for surgery. Br J Anaesth. 2017;118(6):910-917. 
30. Harris PA, Taylor R, Thielke R, Payne J, Gonzalez N, Conde JG. Research electronic data capture (REDCap) - a metadata-driven methodology and workflow process for providing translational research informatics support. J Biomed Inform. 2009;42(2):377-381.

31. Harris PA, Taylor R, Minor BL, et al. The REDCap consortium: Building an international community of software platform partners.J Biomed Inform. 2019;95:103208.

32. Varni JW, Katz ER, Seid M, Quiggins DJ, Friedman-Bender A, Castro CM. The Pediatric Cancer Quality of Life Inventory (PCQL). I. Instrument development, descriptive statistics, and cross-informant variance.J Behav Med. 1998;21(2):179-204.

33. Bhat SR, Goodwin TL, Burwinkle TM, et al. Profile of daily life in children with brain tumors: an assessment of health-related quality of life. J Clin Oncol. 2005;23(24):5493-5500.

34. Meeske K, Katz ER, Palmer SN, Burwinkle T, Varni JW. Parent proxy-reported health-related quality of life and fatigue in pediatric patients diagnosed with brain tumors and acute lymphoblastic leukemia. Cancer. 2004;101(9):2116-2125.

35. Madden JR, Mowry P, Gao D, Cullen PM, Foreman NK. Creative arts therapy improves quality of life for pediatric brain tumor patients receiving outpatient chemotherapy. J Pediatr Oncol Nurs.2010;27(3):133-145.

36. Banks BA, Barrowman NJ, Klaassen R. Health-related quality of life: changes in children undergoing chemotherapy. J Pediatr Hematol Oncol. 2008;30(4):292-297.

37. Klaassen RJ, Krahn M, Gaboury I, et al. Evaluating the ability to detect change of health-related quality of life in children with Hodgkin disease. Cancer. 2010;116(6):1608-1614.

38. McGrath PA. Pain in children: Nature, assessment, and treatment. Scholastic; 1990.

39. Azadinia F, Kamyab M, Behtash H, Ganjavian MS, Javaheri MR. The validity and reliability of noninvasive methods for measuring kyphosis. Clinical Spine Surgery. 2014;27(6):E212-E218.

40. Barrett E, McCreesh K, Lewis J. Intrarater and interrater reliability of the flexicurve index, flexicurve angle, and manual inclinometer for the measurement of thoracic kyphosis. Rehabil Res Pract. 2013;2013:475870.

41. Czaprowski D, Pawlowska P, Gebicka A, Sitarski D, Kotwicki T. Intra- and interobserver repeatability of the assessment of anteroposterior curvatures of the spine using Saunders digital inclinometer. Ortop Traumatol Rehabil. 2012;14(2):145-153.

42. Barrett E, Lenehan B, O'Sullivan K, Lewis J, McCreesh K. Validation of the manual inclinometer and flexicurve for the measurement of thoracic kyphosis. Physiother Theory Pract. 2018;34(4):301-308.

43. IBM SPSS Statistics for Windows [computer program]. Version 27.0. Armonk, NY: IBM Corp.; 2017.

44. Montgomery KE, Raybin JL, Ward J, et al. Using patient-reported outcomes to measure symptoms in children with advanced cancer.Cancer Nurs. 2020;43(4):281-289.

45. Momani TeG, Berry DL. Integrative therapeutic approaches for the management and control of nausea in children undergoing cancer treatment: a systematic review of literature. J Pediatr Oncol Nurs. 2017;34(3):173-184.

46. Thrane S. Effectiveness of integrative modalities for pain and anxiety in children and adolescents with cancer: a systematic review.J Pediatr Oncol Nurs. 2013;30(6):320-332.

47. Coughtrey A, Millington A, Bennett S, et al. The Effectiveness of Psychosocial Interventions for Psychological Outcomes in Pediatric Oncology: A Systematic Review. J Pain Symptom Manage.2018;55(3):10041017.

48. Jacobs SS. Integrative therapy use for management of side effects and toxicities experienced by pediatric oncology patients. Children. 2014;1(3):424-440. 
49. Niedenthal PM, Winkielman P, Mondillon L, Vermeulen N. Embodiment of emotion concepts. J Pers Soc Psychol. 2009;96:1120.

50. Califf RM. Biomarker definitions and their applications. Exp Biol Med (Maywood). 2018;243(3):213-221.

51. Miaskowski C, Aouizerat BE. Biomarkers: symptoms, survivorship, and quality of life. Paper presented at: Seminars in oncology nursing2012.

\section{Hosted file}

Tables.docx available at https://authorea.com/users/427761/articles/531881-associationsbetween-demographics-and-quality-of-life-in-children-in-the-first-year-of-cancer-

treatment 